\title{
Perturbaciones macroeconómicas, movimientos de la tasa de cambio y transmisión sobre precios
}

Recibido: junio 27, 2017 - Aceptado: octubre 26, 2017

Doi: http://dx.doi.org/10.12804/revistas.urosario.edu.co/economia/a.6455

\author{
Hernán Rincón-Castro* \\ Norberto Rodríguez-Niño ${ }^{+}$ \\ John Castro-Pantoja ${ }^{\ddagger}$
}

\section{Resumen}

La literatura que ha estudiado la transmisión de los movimientos de la tasa de cambio sobre los precios - exchange rate pass-through - asume que aquellos son exógenos a las perturbaciones que impactan la economía. Este supuesto ha sido revaluado a partir de modelos macroeconómicos modernos, que muestran que dichos movimientos son endógenos. Basado en este resultado, el presente documento muestra evidencia de que efectivamente el grado de transmisión depende de la naturaleza de la perturbación que origine el movimiento cambiario, es decir, que la transmisión es perturbación-dependiente (shock-dependent).

Palabras clave: perturbaciones, tasa de cambio, transmisión sobre precios, restricciones de signo, SVAR.

Clasificación JEL: E32, F31, E31, E52, C51

Los autores agradecen los valiosos comentarios de Hernando Vargas y de los dos evaluadores anónimos. Las opiniones, errores u omisiones contenidos en este documento son responsabilidad exclusiva de los autores y no comprometen al Banco de la República ni a su Junta Directiva.

* Investigador Principal de la Unidad de Investigaciones, Banco de la República. Correspondencia. Correo electrónico: hrincoca@banrep.gov.co.

+ Econometrista Principal del Departamento de Modelos Macroeconómicos, Banco de la República.

$\ddagger$ Estudiante en Práctica, Banco de la República.

Cómo citar este artículo: Rincón-Castro, H., Rodríguez-Niño, N., \& Castro-Pantoja, J. (2017). Perturbaciones macroeconómicas, movimientos de la tasa de cambio y transmisión sobre precios. Revista de Economía del Rosario, 20(2), 213-241.

Doi: http://dx.doi.org/10.12804/revistas.urosario.edu.co/economia/a.6455 


\title{
Macroeconomic Shocks, Movements of the Exchange Rate and Pass-Through on Prices
}

\begin{abstract}
The literature that has studied the transmission of exchange rate movements on prices, known as exchange rate pass-through, assumes that they are exogenous to the shocks that impact the economy. This assumption has been reviewed according to predictions of modern macroeconomic models, which point out that exchange movements are endogenous. Based on this prediction, this paper shows that the degree of transmission depends on the type of shock that gives rise to the exchange rate movement, i.e., transmission is shock-dependent.
\end{abstract}

Keywords: Macroeconomic shocks, exchange rate, pass-through on prices on prices, sign restrictions, SVAR.

JEL classification: E32, F31, E31, E52, C51

\section{Perturbações macroeconómicas, movimentos da taxa de câmbio e transmissão sobre preços}

Resumo

A literatura que tem estudado a transmissão dos movimentos da taxa de câmbio sobre os preços -exchange rate pass-through- assume que aqueles são exógenos às perturbações que impactam a economia. Este suposto tem sido reavaliado a partir de modelos macroeconómicos modernos, que mostram que ditos movimentos são endógenos. Baseado neste resultado, o presente documento mostra evidência que efetivamente o grau de transmissão depende da natureza da perturbação que origine o movimento cambiário, é dizer, que a transmissão é perturbação-dependente (shock-dependent).

Palavras-chave: perturbações, taxa de câmbio, transmissão sobre preços, restrições de signo, SVAR.

Classificação JEL: E32, F31, E31, E52, C51

\section{Introducción}

Las perturbaciones macroeconómicas externas e internas que enfrenta una economía impactan sus principales variables macroeconómicas, entre ellas su moneda y sus precios. De ahí, la importancia de su análisis para las autoridades económicas, en particular para las monetarias.

Los objetivos del documento son, en primer lugar, estimar el grado de transmisión de los movimientos endógenos de la tasa de cambio - exchange rate pass-through - sobre el índice de precios al consumidor (IPC), para una 
economía pequeña y abierta. A diferencia de casi toda la literatura internacional, y de investigaciones anteriores realizadas por los autores y la literatura local, el presente estudio asume que las variaciones de la tasa de cambio provienen de perturbaciones macroeconómicas en variables fundamentales externas e internas, las cuales se identifican de manera independiente a partir de las predicciones de modelos DSGE, tal como lo hacen Shambaugh (2008) y Forbes et al. $(2015)^{1}$. Por tanto, en este documento el grado de transmisión de movimientos de la tasa de cambio sobre los precios es perturbación-dependiente (shock-dependent) ${ }^{2}$. En segundo lugar, el objetivo también es descomponer históricamente las variaciones de la tasa de cambio y la inflación durante el periodo de estudio, con el fin de identificar las perturbaciones que originaron dichas variaciones y cuantificar su participación.

Con el fin de cumplir con los objetivos planteados, se utilizan datos mensuales de Colombia y sus principales socios comerciales - para los propósitos de este documento, se asume que Estados Unidos representa al resto del mundo- El periodo de estudio va desde enero de 2001 hasta junio de 2016 y el modelo econométrico es un VAR estructural (SVAR) lineal estimado por máxima verosimilitud. Recuérdese que un VAR es una forma reducida que resume los datos; mientras que un SVAR "proporciona una interpretación de los datos" (Fry \& Pagan, 2011, p. 938). La presentación de los resultados se asemeja a la de Forbes et al. (2015).

El documento se divide en cuatro secciones. La primera sección describe el modelo econométrico e identifica las distintas perturbaciones, a partir de la teoría económica que se referencia. La segunda muestra y estudia las funciones de impulso, respuestas estimadas y calcula el grado de la transmisión de las variaciones de la tasa de cambio de sobre el IPC. La tercera presenta y analiza las descomposiciones históricas (HDs) de las perturbaciones para la variación anual de la tasa de cambio y la inflación. Estas permiten estimar la participación de cada una ellas en la explicación de los movimientos de las variables endógenas mencionadas, para cada momento del tiempo durante la muestra. La cuarta sección resume las principales conclusiones.

1 Para una revisión exhaustiva de la literatura local e internacional sobre la transmisión de los movimientos de la tasa de cambio sobre los precios, véase Rincón-Castro y Rodríguez-Niño (2016).

2 Así, este documento complementa a Rincón-Castro y Rodríguez-Niño (2016), quienes muestran, entre otros, que la transmisión es estado-dependiente (state-dependent). 


\section{Modelo econométrico e identificación de las perturbaciones}

\subsection{Modelo econométrico}

El modelo SVAR se define por el siguiente vector:

$$
Y_{t}=\left[\begin{array}{c}
\pi_{t}^{I P C} \\
\Delta e_{t} \\
\Delta p i b r \\
T I B_{t} \\
V I X_{t} \\
\Delta W T I \\
\Delta p i b r_{t}^{*}
\end{array}\right]
$$

donde $\pi^{I P C}$ representa la variación porcentual anual del índice de precios al consumidor (IPC), e es la tasa cambio nominal efectiva del peso, ponderada por el comercio total proveniente de los principales socios comerciales del país, pibr es el producto interno bruto (РІB) real de Colombia, TIB es la tasa de interés interbancaria (el instrumento operativo de política monetaria interna), VIX es una medida del riesgo en los mercados financieros internacionales, WTI es el precio real del petróleo West Texas Intermediate y pibr* es el PIB del resto del mundo. Todas las variables, excepto la tasa de interés y el viX, son previamente transformadas a logaritmos naturales. $\Delta$ es el operador de diferencias de orden 12, de tal manera que las respectivas variables se expresan en tasas de crecimiento porcentual anual. Las series, sus fuentes y las notas metodológicas se describen en el apéndice A.1.

El vector de perturbaciones estructurales se define como:

$$
\boldsymbol{\varepsilon}_{t}=\left[\begin{array}{c}
\varepsilon_{t}^{\text {Demanda }} \\
\varepsilon_{t}^{\text {Oferta }} \\
\varepsilon_{t}^{\text {Tasa de cambio }} \\
\varepsilon_{t}^{\text {Pol. }} \text { monetaria } \\
\\
\varepsilon_{t}^{\text {VIX }} \\
\varepsilon_{t}^{\text {Precio petróleo }} \\
\\
\varepsilon_{t}^{\text {PIB }}
\end{array}\right]
$$

Se asume que las perturbaciones son independientes y no anticipadas. La perturbación de la demanda interna $\left(\varepsilon_{t}^{\text {Demanda }}\right)$ se origina en una perturbación positiva de las preferencias o el ingreso disponible de los hogares, por ejemplo, 
por un subsidio público; de la oferta interna $\left(\varepsilon_{t}^{\text {Oferta }}\right)$, en un aumento en la productividad; de la tasa de cambio ( $\varepsilon_{t}^{\text {Tasa de cambio }}$, en un aumento exógeno del riesgo país, que produce una perturbación del peso, por ejemplo, por un aumento del riesgo soberano; de la política monetaria interna ( $\left.\varepsilon_{t}^{\text {Pol. monetaria }}\right)$, en un aumento de la tasa de interés de política; del riesgo externo $\left(\varepsilon_{t}^{V I X}\right)$, en un aumento de la turbulencia en los mercados financieros internacionales; del precio internacional del petróleo $\left(\varepsilon_{t}^{\text {Precio petróleo }}\right)$, resultado de presiones del mercado, que no se identifican si son consecuencia de choques de oferta o de demanda externos, como se justificará más adelante, y de cambios no anticipados del PIB del resto del mundo $\left(\varepsilon_{t}^{P I B^{*}}\right)$.

Ahora, el grado de transmisión de los movimientos de la tasa de cambio sobre el IPC hasta el momento $\tau$ (o coeficiente de pass-through), se define como la mediana de la razón de las funciones de impulso respuesta acumuladas del IPC y de la tasa de cambio ante la perturbación estructural $i$-ésima (ver Rincón-Castro \& Rodríguez-Niño, 2016), así:

$$
P T_{\tau}^{I P C}=\frac{\sum_{j=0}^{\tau} \frac{\partial \pi_{t+j}^{I P C}}{\partial \varepsilon_{t}^{i}}}{\sum_{j=0}^{\tau} \frac{\partial \Delta e_{t+j}}{\partial \varepsilon_{t}^{i}}}
$$

$i=$ Demanda,Oferta,Tasa de cambio,Pol.monetaria,VIX,Precio petróleo,PIB externo

El lector debe notar que esta definición del grado de transmisión cambiaria no es estándar en la literatura internacional o local, que lo interpreta simplemente como el coeficiente de la tasa de cambio en una ecuación de precios. La literatura asume que los movimientos de la tasa de cambio son exógenos y que la transmisión es constante en el tiempo y no depende del estado de la economía, de las perturbaciones macroeconómicas u otras variables. Por esta razón, se le invita a que esté atento a este cambio y regrese a revisar la definición (3), cuando se presenten los resultados.

\subsection{Identificación del sistema SVAR}

La identificación de las perturbaciones utiliza una combinación de restricciones de ceros en los parámetros de corto y largo plazo, y restricciones de signo derivadas de las predicciones de modelos Neokeynesianos DSGE, en particular, de los desarrollados por Gali (1999), Blanchard y Gali (2007) y Bonaldi et al. (2010); así como de los modelos empíricos de Blanchard y Quah (1989) y Kilian 
$(2009)^{3}$. Por otro lado, las estimaciones econométricas utilizan los algoritmos derivados y analizados por Rubio-Ramírez et al. (2010), para modelos exactamente identificados; y por Binning (2013), para modelos sub-identificados.

Como es bien conocido en la literatura, la identificación de las perturbaciones es un paso fundamental para darle estructura y soporte teórico a los modelos de series de tiempo, como el aquí planteado, y a sus resultados. Además, desde un punto de vista empírico, una buena identificación permite enfrentar críticas a los modelos recursivos como el utilizado (ver, por ejemplo, aquellas de Faust \& Rogers 2003). En adición, se garantiza que los resultados sean robustos y libres de los inconvenientes originados en un ordenamiento arbitrario de las variables incluidas en el sistema VAR.

Antes de continuar, es necesario anotar que el régimen monetario vigente en Colombia para la muestra analizada es de inflación objetivo (meta anual de $3 \% \pm 1 \%$ ). El régimen cambiario es de flotación. Se supone que el banco central sigue una regla de política tipo Taylor.

Los supuestos más importantes para la identificación del sistema SVAR definido son:

(1) En el corto plazo, como lo predicen los modelos referenciados, las alteraciones de la demanda afectan el producto, y no lo contrario.

(2) Las perturbaciones de la oferta son las únicas que afectan el producto en el largo plazo.

(3) Las perturbaciones de las variables internas no afectan a las variables externas a ningún plazo (se asume que Colombia es una economía "pequeña") ${ }^{4}$.

(4) Las alteraciones de la oferta del resto del mundo tienen múltiples canales de transmisión, por lo que no es posible independizar las perturbaciones y los efectos particulares sobre cada una de las variables endógenas. Como consecuencia, estas se suponen libres de cualquier restricción.

(5) La perturbación del precio del petróleo tiene un impacto positivo sobre el producto. Dos razones justifican este supuesto. Primero, Colombia es un

3 La identificación de modelos VAR por medio de restricciones de signo, derivadas de modelos teóricos microfundamentados, fue introducida por Canova y De Nicolo (2002). Una excelente revisión de la literatura sobre sobre este aspecto se encuentra en Fry y Pagan (2011).

4 Este supuesto implica, desde el punto de vista económico, que Colombia es tomador de precios en el mercado mundial de bienes y de capitales. Por otro lado, desde el punto de vista econométrico, que se imponen explícitamente restricciones de exogeneidad fuerte sobre las variables externas, es decir, se obliga a que los coeficientes de todos los rezagos de las variables internas en todas las demás ecuaciones sean iguales a cero. Esto ayudó a que las funciones de impulso respuesta se comportaran acorde con la teoría y la intuición económica. 
país productor y exportador de productos básicos, altamente influenciado por el comportamiento de sus precios —efecto términos de intercambio-, así como por sus cantidades —efecto encadenamientos productivos ${ }^{5}$ De acuerdo con estadísticas oficiales, al finalizar 2013, año anterior al derrumbamiento extraordinario y persistente de sus precios, el petróleo representaba el $5 \%$ del PIB y el 55 \% de las exportaciones totales del país. Así mismo, solo dicho sector le reportó rentas al gobierno del orden del $20 \%$ de sus ingresos totales. Segundo, dada la dificultad de identificar las fuentes de sus alteraciones, y las distintas consecuencias macroeconómicas que pueden generar cada una de ellas, como lo muestra Kilian (2009), simplemente se asume que su impacto es positivo sobre el producto ${ }^{6}$.

La tabla 1 resume las restricciones que se imponen sobre el sistema SVAR, con el fin de identificar cada una de las perturbaciones. Los símbolos "+" ("-") del cuadro indican que la perturbación i-ésima afecta positivamente (negativamente) el valor acumulado de la respectiva variable endógena, al final del primer año. En el mediano y largo plazo, el número “ 0 ” indica que la perturbación $i$-ésima no tiene efecto sobre las variables 'endógenas' externas (supuesto de economía "pequeña"). El “ 0 " también indica que ni la demanda, ni las perturbaciones nominales ni el VIX afectan el PIB real en el largo plazo. Los espacios en blanco señalan que no hay predicción alguna del impacto sobre la variable endógena, por tanto no se impone ninguna restricción (se estiman libremente) ${ }^{7}$.

5 Un estudio comprensivo sobre el papel del sector petrolero en la economía colombiana se encuentra en López et al. (2013).

6 Nótese que las alteraciones pueden originarse en movimientos de la oferta o demanda externas o por motivos financieros. La primera por un aumento en la producción local e internacional y la segunda por un aumento en la demanda mundial, por ejemplo, por la mayor demanda de países emergentes grandes y por relajamiento de la política monetaria internacional, entre otros. La tercera por las variaciones del valor del dólar (USD), la moneda en que se fijan los precios del petróleo (si se deprecia, el precio del petróleo aumenta, si se aprecia, cae).

7 ¿En la práctica, cómo funciona el proceso de identificación? Una vez se imponen las restricciones de ceros, se evalúa primero si el modelo está sobreidentificado. Si lo está, no se puede proceder con las restricciones de signo. Si no lo está, se procede a rotar aleatoriamente la descomposición de Cholesky de la matriz de varianzas y covarianzas de los residuos del modelo estimado. Cada rotación se obtiene por medio de la descomposición "QR" de una matriz aleatoria normal. El lector interesado en conocer en qué consiste dicha descomposición y cómo se deriva cuando se imponen restricciones de exogeneidad fuerte, como las del presente documento, puede consultar a Ouliaris et al. (2016). Con la matriz de rotación $Q R$ se generan nuevas funciones de impulso respuesta y se evalúa si se cumplen las restricciones de signo. Las matrices generadas que no cumplan todas las restricciones son descartadas y se repite el procedimiento anterior. Las matrices que cumplen las restricciones 
Tabla 1. Restricciones sobre el sistema SVAR

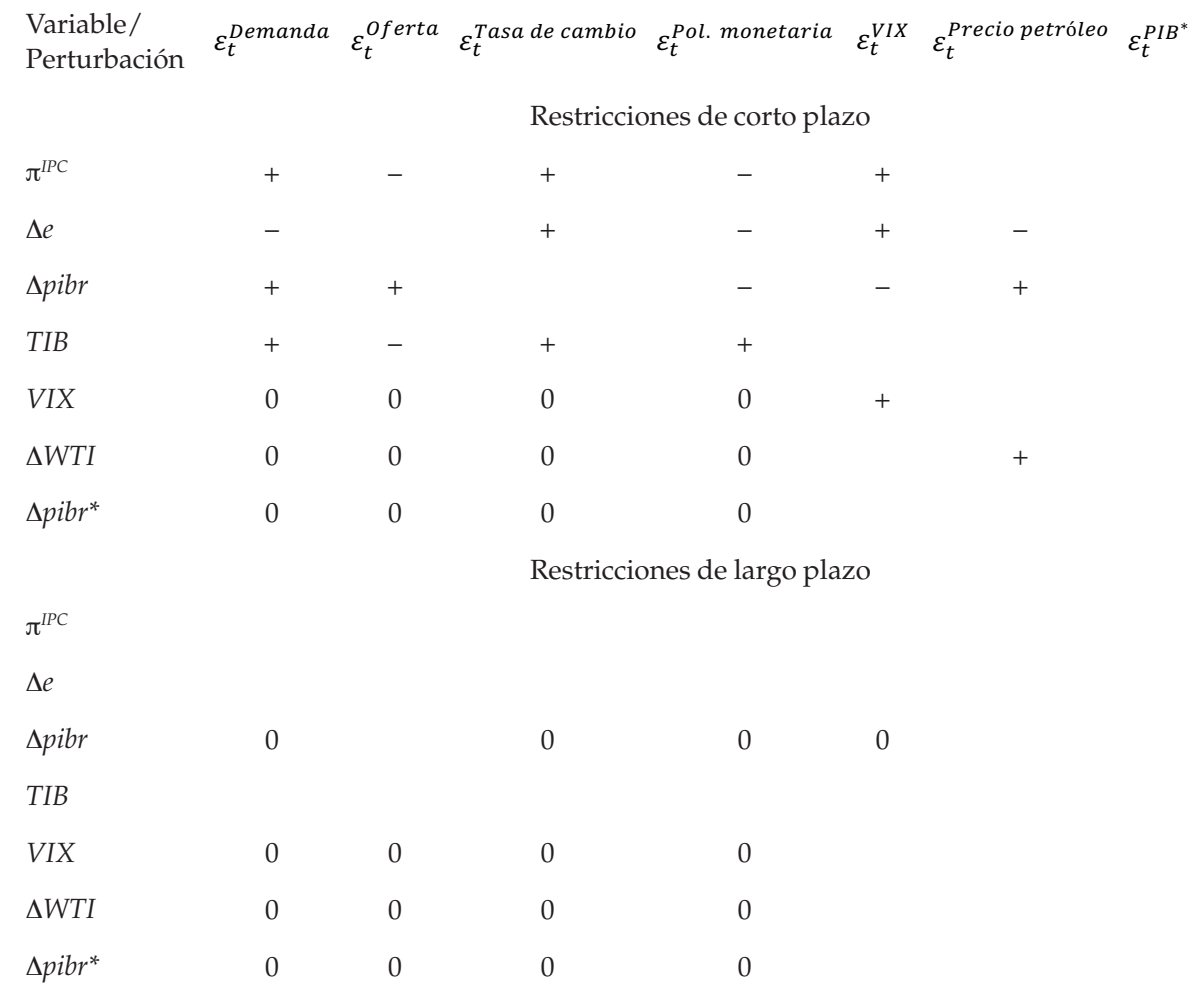

Fuente: inferencias propias a partir de la literatura referenciada.

Nota: se agradecen las valiosas sugerencias realizadas por Diego Rodríguez, Investigador del Departamento de Modelos Macroeconómicos del Banco de la República.

La narrativa sobre los canales de transmisión de cada una de las perturbaciones se realiza de manera esquemática, como sigue.

1. Perturbación de la demanda interna, resultado de cambios en las preferencias o aumentos en el ingreso disponible, que se manifiestan en un aumento del gasto agregado $\Rightarrow$ demanda agregada $\uparrow \Rightarrow \Delta$ pibr $\uparrow \Rightarrow$ demanda por factores de producción $\uparrow \Rightarrow$ demanda de trabajo $\uparrow \Rightarrow$ salario real $\uparrow \Rightarrow$ $\pi^{I P C} \uparrow \Rightarrow$ TIB $\uparrow$. Por su lado, y de acuerdo con la paridad descubierta de intereses, el aumento de la tasa de interés interna genera un aumento en las expectativas de depreciación (se supone que la tasa de interés externa

se almacenan. Esta rutina se repite hasta que el número de matrices almacenadas sea igual a las iteraciones requeridas. 
y el riesgo país permanecen constantes), que requieren una apreciación inicial de la moneda local $\Rightarrow \Delta \mathrm{e} \downarrow$.

2. Perturbación de la oferta interna, por un cambio positivo en la productividad total de los factores $\Rightarrow$ costo marginal $\downarrow \Rightarrow \Delta$ pibr $\uparrow \Rightarrow \pi^{I P C} \downarrow$, ya que el PIB potencial aumenta relativamente más que el observado y la brecha del producto se torna negativa $\Rightarrow$ TIB $\downarrow$. El efecto agregado sobre la tasa de cambio es incierto.

3. Perturbación cambiaria autónoma, ante aumento en el riesgo país $\Rightarrow$ expectativas de apreciación $\uparrow$ (se supone que la tasa de interés externa permanece constante), que requieren una depreciación inicial de la moneda local $\Rightarrow \Delta \mathrm{e} \uparrow \Rightarrow$ precio de los bienes importados y al productor aumentan. Este es el canal directo de transmisión de los movimientos de la tasa de cambio sobre los precios (se supone que los precios externos permanecen constantes). Entonces, $\pi^{I P C} \uparrow$ y la TIB $\uparrow$. El efecto agregado sobre el PIB es incierto.

4. Perturbación positiva de la política monetaria $\Rightarrow$ ТІв $\uparrow \Rightarrow$ demanda agregada $\downarrow \Rightarrow \Delta$ pibr $\downarrow \Rightarrow \pi^{I P C} \downarrow$. Por su lado, y de acuerdo con la paridad descubierta de intereses, el aumento de la tasa interna genera un aumento en las expectativas de depreciación (se supone que la tasa de interés externa y el riesgo país permanecen constantes), que requieren una apreciación inicial de la moneda local $\Rightarrow \Delta \mathrm{e} \downarrow$.

5. Perturbación del riesgo externo: las variables endógenas $\Delta \mathrm{e}$ y $\pi^{I P C}$ presentan los mismos signos de la perturbación cambiaria, pero se supone que el efecto sobre el PIB es negativo. Esto podría ser explicado por una salida de capitales, motivado en un comportamiento tipo "home bias" (preferencia por las inversiones en el país de origen de los capitales), o por la reducción del apetito por riesgo de los inversionistas internacionales, que se manifiesta en una disminución de la oferta financiamiento externo del consumo y la inversión locales; por tanto, $\Delta$ pibr $\downarrow$. El efecto agregado sobre la TIB es incierto.

6. Perturbación positiva del precio del petróleo_ $\Rightarrow$ Precio del petróleo $\uparrow \Rightarrow$ entran al país ingresos petroleros que aprecian el peso $(\Delta \mathrm{e} \downarrow)$. Como el sector petrolero también genera $\mathrm{PIB} \Rightarrow \Delta$ pibr $\uparrow$. El efecto agregado sobre la $\pi^{I P C}$ y la тів es incierto.

7. Perturbación del PIB externo: el efecto agregado sobre las variables internas y externas es incierto. 


\section{Estimaciones}

En esta sección se presentan y analizan las estimaciones. Se advierte de nuevo que cada una de las restricciones de signo se acumula y se hace efectiva hasta un año después de cada perturbación ${ }^{8}$. Esto permite guardar consistencia entre las estimaciones, las predicciones teóricas que identifican el sistema y los rezagos propios de los impactos de las distintas perturbaciones sobre las variables endógenas ${ }^{9}$.

Los estadísticos y pruebas previas requeridas por las estimaciones no se presentan y están a disposición. De acuerdo con ellas, el sistema SVAR estimado es estacionario. También están disponibles las distintas pruebas de robustez realizadas con restricciones de identificación y con variables internas y externas alternativas, como, por ejemplo, con distintas agregaciones de los precios de los bienes básicos, o con distintas medidas de la actividad real o de los riesgos internos y externos. Por último, es pertinente informar que la escritura de los códigos y su implementación fueron realizadas en Matlab.

\subsection{Funciones de impulso respuesta de las variables endógenas}

La figura 1 muestra las medianas de las 10000 funciones de impulso respuesta obtenidas, mediante tal número de iteraciones del algoritmo de estimación, para cada una de las variables endógenas del sistema y ante cada perturbación. Las respuestas, excepto para la TIB, son acumuladas, de tal manera que las figuras muestran el impacto en el tiempo sobre el nivel de cada variable. Las áreas sombreadas corresponden a los intervalos de confiabilidad del $68 \%$ y $90 \%{ }^{10}$. Con el fin de permitir la comparación de los resultados de la transmisión cambiaria ante las distintas perturbaciones, las funciones de impulso respuesta se estandarizan; de tal manera que a los doce meses las perturbaciones generan una variación de la tasa de cambio del $1 \%$, tal y como lo hacen Shambaugh (2008) y Forbes et al. (2015). Las funciones de impulso respuesta de las variables endógenas a sus propias perturbaciones, y que no son mostradas en la figura 1, se presentan en el apéndice A.2.

8 La excepción es el impacto de la perturbación del riesgo externo sobre variación de la tasa de cambio, que solo se restringe por dos periodos, de acuerdo con la naturaleza financiera de la variable; responde más rápidamente que en el caso de variables reales.

9 Fry y Pagan (2011) muestran por qué podría ser válido imponer restricciones de signo en un orden superior al de los rezagos del modelo, como se realiza aquí.

10 Los intervalos de las respuestas ante una perturbación de la oferta interna son obtenidos por Bootstrapping. Esto se debe a que no se obtiene variabilidad cuando se imponen las restricciones descritas en el texto, como sucede en algunos estudios de la literatura relacionada, por ejemplo, en Binning (2013). 
Las figuras muestran resultados que son, en general, estadísticamente significativos y consistentes con las restricciones que identifican el sistema. Por ejemplo, una perturbación a la demanda interna aumenta el PIB y, como respuesta, aumenta la inflación y la tasa de interés de política. Esto conlleva una apreciación de la moneda. En el caso de la perturbación de la política monetaria, la inflación disminuye, el peso se aprecia y el PIB disminuye, así sea en menor grado, como se espera. Cuando se presenta una perturbación del riesgo externo, la inflación y la tasa de cambio aumentan, aunque esta última lo hace al impacto y luego se reduce de manera acelerada. Por su lado, el PIB se reduce al impacto y luego aumenta rápidamente hacia su nivel de largo plazo. Como en el caso de la perturbación de la demanda, pareciera que esta domina el comportamiento de la tasa de cambio en el mediano plazo. Por su lado, la política monetaria se vuelve restrictiva ante el aumento de la inflación.

Cuando ocurre una perturbación del precio del petróleo, aumenta el PIB y se aprecia el peso, como predice la teoría y se observa en la práctica. Sin embargo, los datos identifican un leve aumento de la inflación, a pesar de la apreciación. Esto significa que el efecto ingreso proveniente de la perturbación petrolera sobre la demanda agregada interna compensa el efecto deflacionario de la apreciación. La tasa de interés de política reacciona y aumenta ante el incremento de la inflación.

Figura 1. Funciones de impulso respuesta

Ante perturbación de la demanda interna
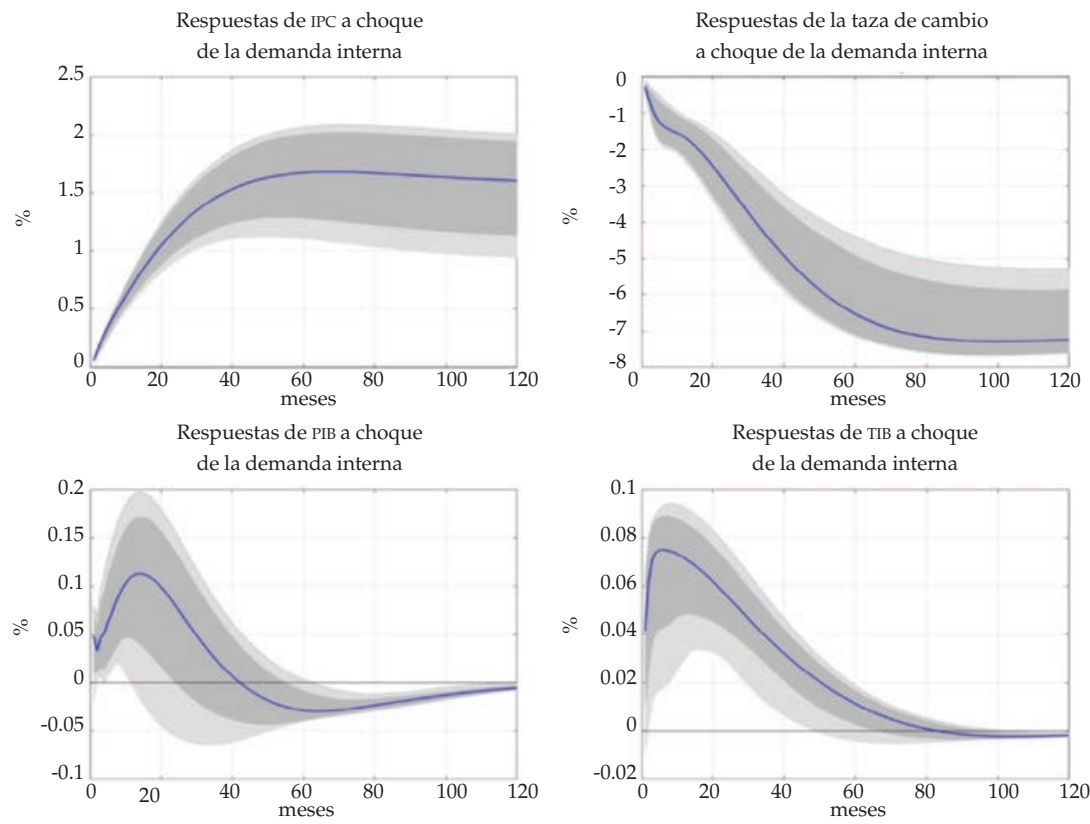
Ante perturbación de la oferta interna
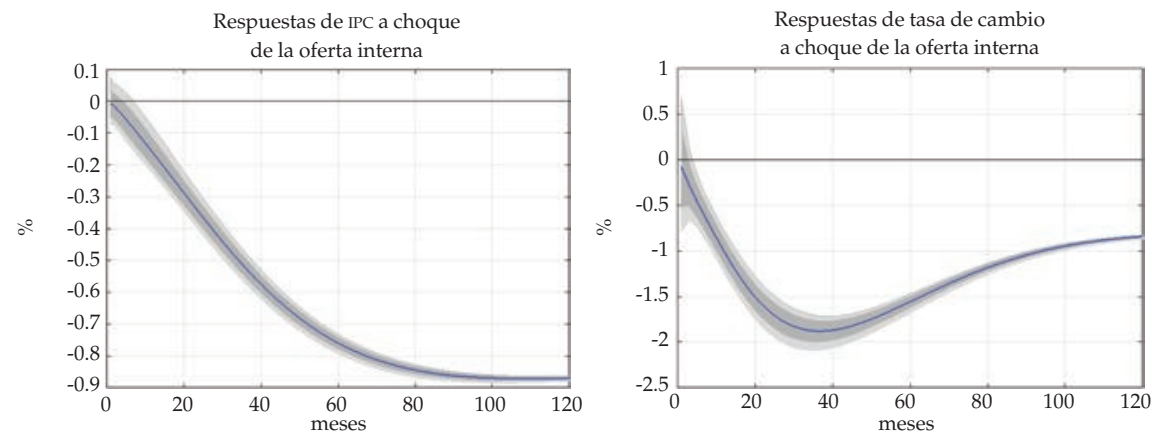

Respuestas del PIB
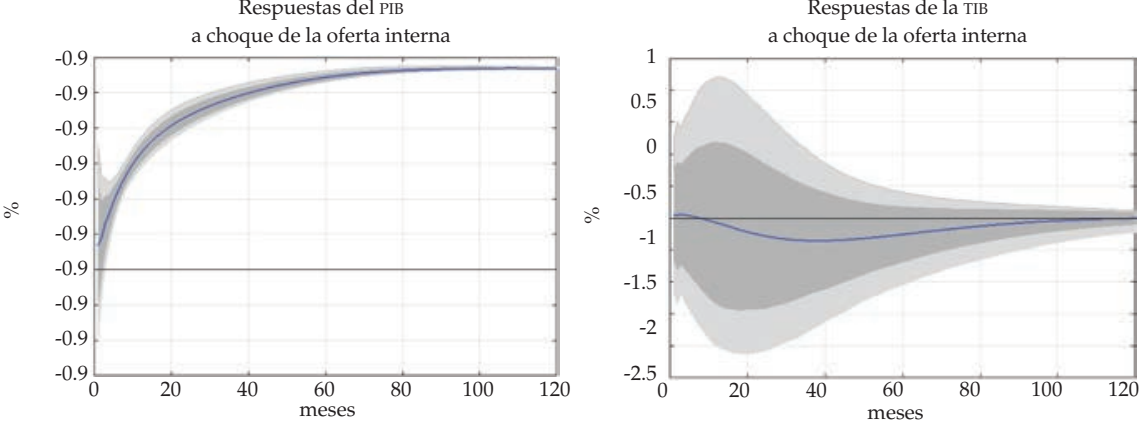

Ante perturbación de la tasa de cambio
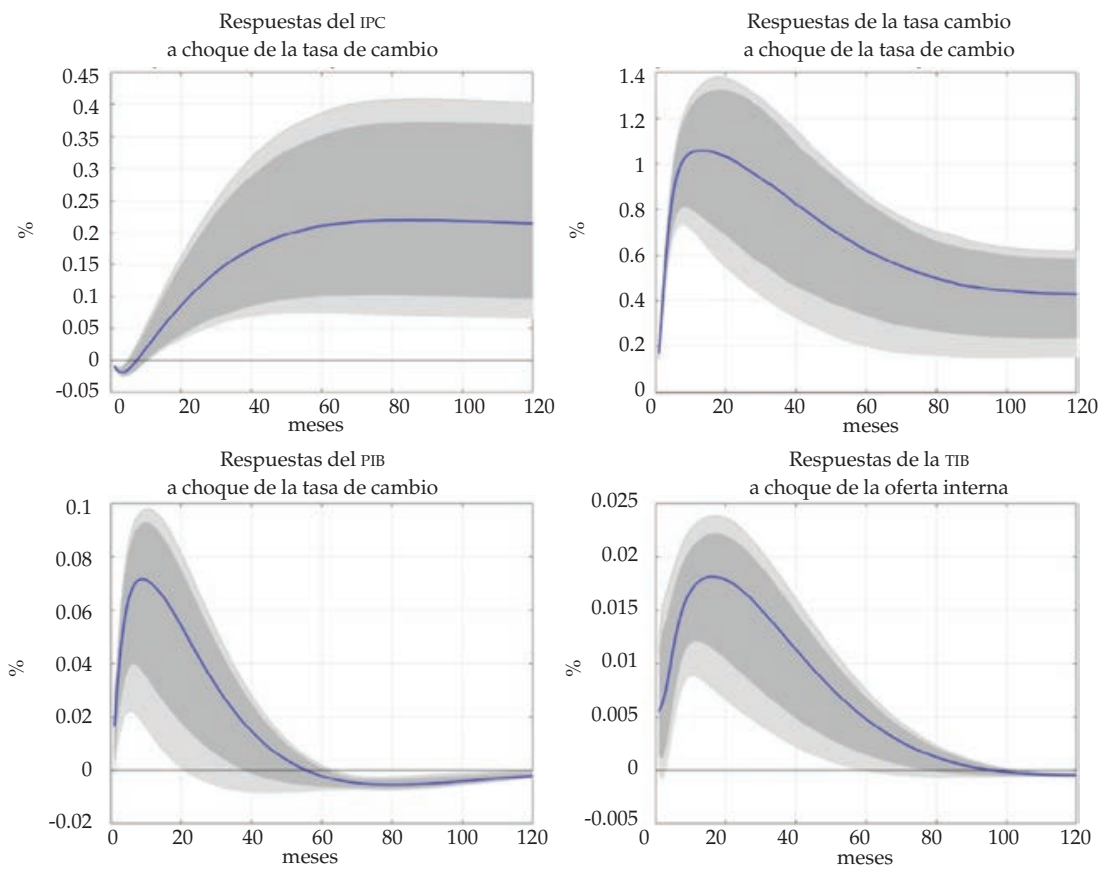
Ante perturbación de la política monetaria
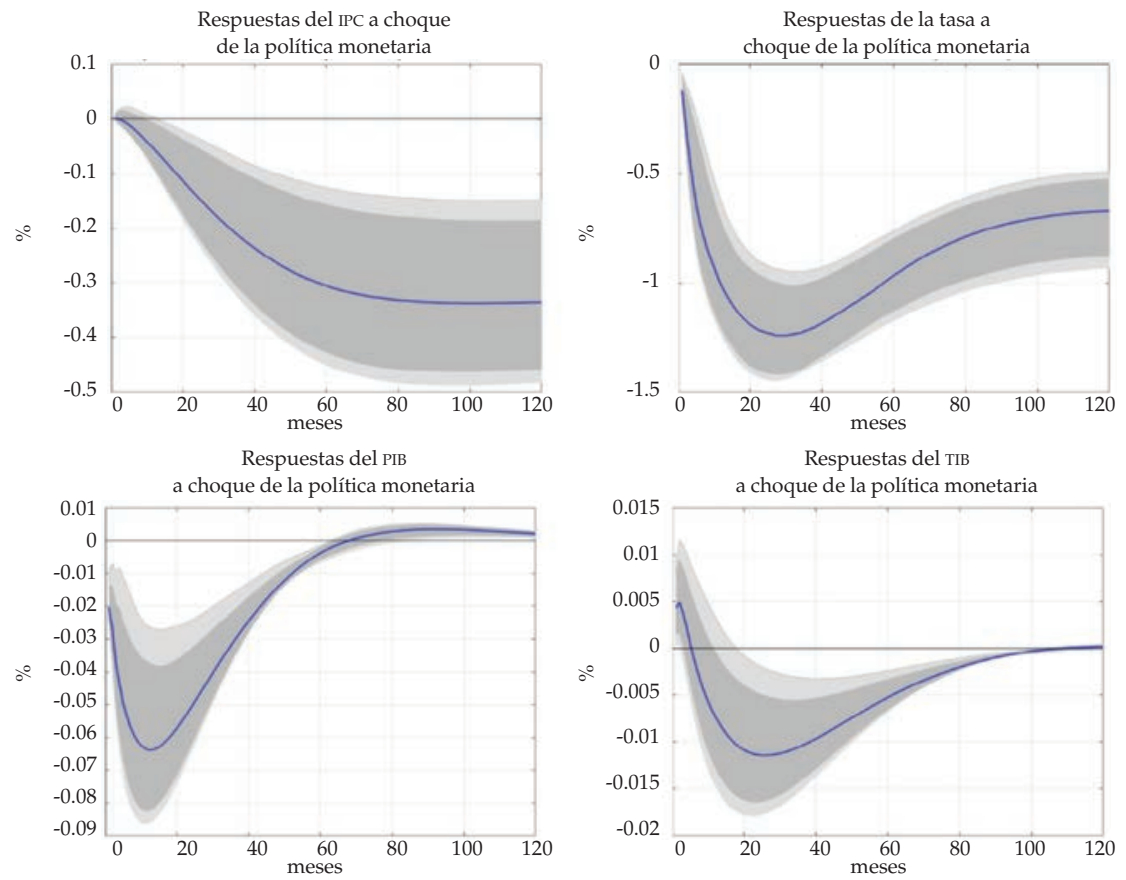

Ante perturbación del riesgo externo (VIX)
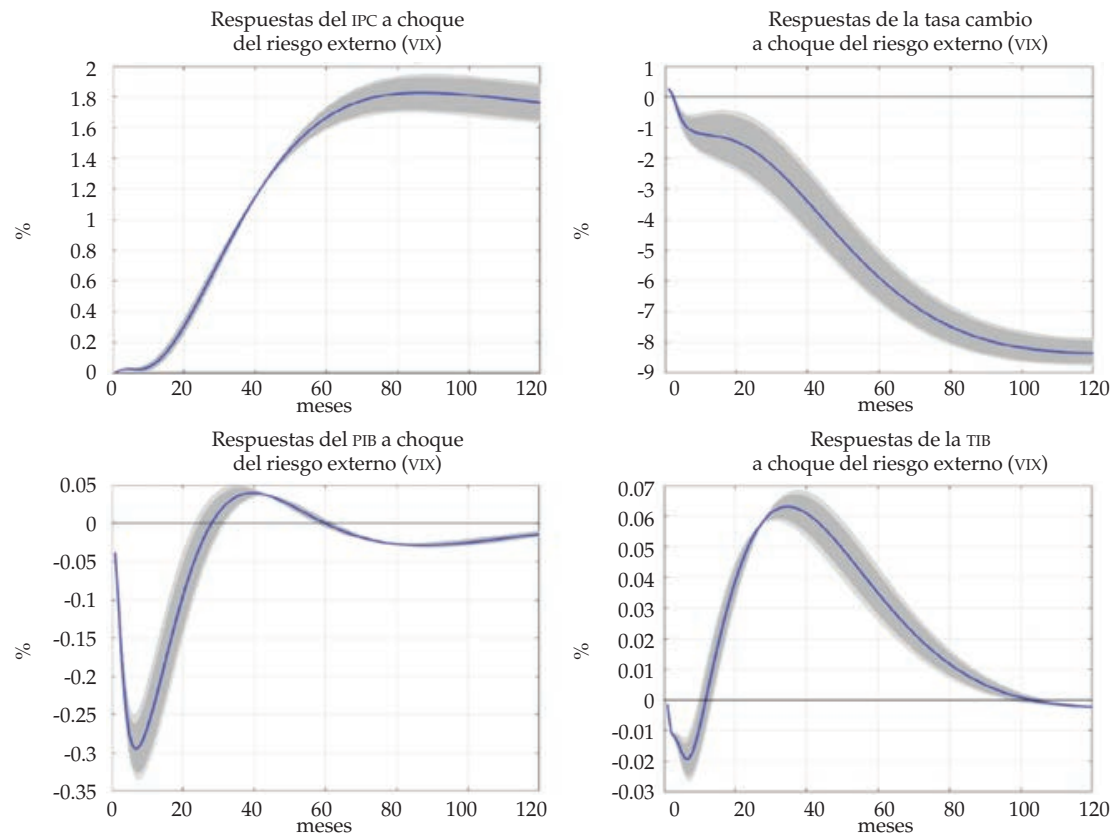

Ante perturbación del precio del petróleo 

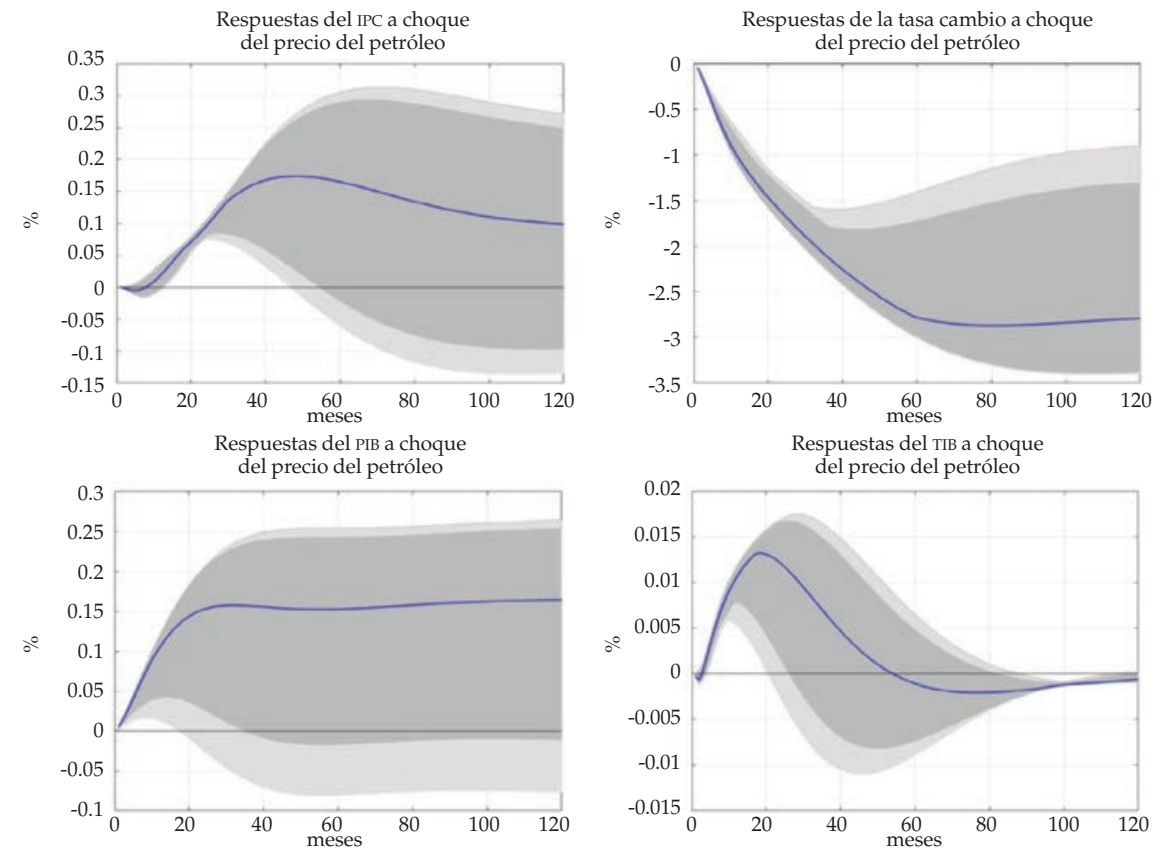

Ante perturbación del РІВ externo

Respuestas del IPC a choque del del PIB externo
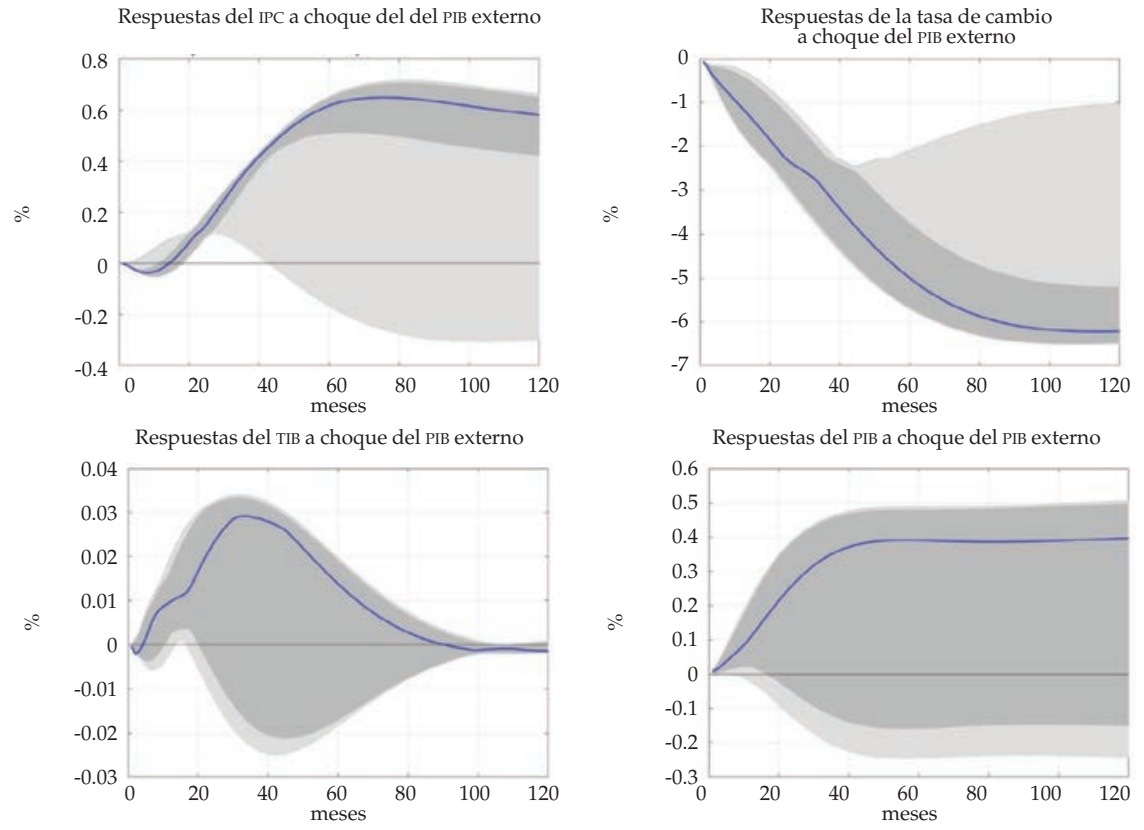

Fuente: cálculos de los autores. 
Una pregunta inmediata que surge del impacto estimado de la perturbación del precio del petróleo es la siguiente: ¿cómo se pueden interpretar la histórica depreciación del peso y la alta inflación local observadas entre 2014 y 2016 a la luz de este ${ }^{11}$ ¿Se podría decir que el efecto inflacionario de la fuerte depreciación se hubiera anulado, o por lo menos aminorado significativamente, si la demanda hubiera caído más rápidamente, por ejemplo, por una reacción más rápida y fuerte de política monetaria? De acuerdo con las estimaciones, la respuesta es sí. Hay que señalar, sin embargo, que el presente modelo de regresión es lineal y no captura posibles no linealidades presentes en los datos, como lo muestran Rincón-Castro y Rodríguez-Niño (2016), y que podrían afectar los resultados de esta investigación. Así, estas podrían haber actuado y, ante la fuerte depreciación, sin precedentes recientes, la inflación originada ante tal perturbación hubiera aumentado de todos modos.

Por último, cuando se presenta una perturbación del PIB externo, el PIB local aumenta, probablemente motivado en un aumento de las exportaciones, la tasa de cambio se aprecia y la inflación cae al impacto. La autoridad monetaria reacciona al impacto y reduce la tasa de interés de política; que luego aumenta rápidamente, ante el aumento de la inflación producida por el crecimiento de la demanda agregada interna.

Ahora, las funciones de impulso respuesta estimadas se utilizan en la siguiente subsección para el cálculo del grado de transmisión cambiaria sobre IPC, ante cada una de las perturbaciones.

\subsection{Estimación del grado de transmisión de los movimientos de la tasa de cambio sobre el IPC}

De acuerdo con la ecuación (3), el coeficiente de transmisión de la perturbación $i$-ésima es la mediana de la razón de las funciones de impulso respuesta acumuladas del IPC y de la tasa de cambio del peso, que se mostraron en la figura 1. Dicha razón es calculada, para cada una de las 10000 funciones de impulso respuesta estimadas, mediante tal número de iteraciones del algoritmo de estimación. Por tanto, la figura 2 muestra la transmisión acumulada entre el momento $t=1$ de la perturbación $i$-ésima y hasta 120 meses. Al igual que en la figura anterior, las áreas sombreadas corresponden a los intervalos de confiabilidad del $68 \%$ y $90 \%$.

En primer lugar, ante una perturbación de la demanda la transmisión es negativa, en grados del $20 \%$ al impacto y del $40 \%$ en un lapso de menos de

11 Entre julio de 2014 y febrero de 2016 el peso se deprecio $60 \%$ y el IPC aumento 10,5\%. 
dos años. Luego se reduce lentamente en el tiempo y converge al $20 \%$ en el largo plazo. ¿Esto qué quiere decir en términos del nivel de precios? Lo que quiere decir es que ante una perturbación de demanda, el IPC debería reducirse $20 \%$ de la apreciación al impacto, $40 \%$ en menos de dos años y $20 \%$ en el largo plazo. Sin embargo, de acuerdo con las funciones de impulso respuesta, ante tal perturbación la inflación acumulada aumenta. ¿Entonces, qué es lo que sucede? Que el impacto positivo de dicha perturbación sobre el IPC, más que compensa el efecto negativo de la apreciación, por este motivo el IPC aumenta. La respuesta de política monetaria es menor.

En contraste, cuando ocurre una perturbación de la oferta, la transmisión es positiva en grados del $13 \%$ y $21 \%$ hasta uno y dos años, respectivamente. En el largo plazo, la transmisión acumulada sigue aumentando y converge al $100 \%$, luego de transcurridos 120 meses. Ahora, ¿qué sucede con el nivel de precios? Disminuye, como lo muestra la función de impulso respuesta, en los porcentajes de la apreciación mencionados. Al contrario de la perturbación de la demanda, la de oferta retroalimenta los efectos negativos de la apreciación sobre los precios, de tal manera que ellos caen. El tamaño de la respuesta de la política monetaria es nulo estadísticamente, como lo muestra el intervalo de confiabilidad.

Ante un choque exógeno o autónomo de la tasa de cambio, la transmisión es positiva en grados cercanos a cero al impacto, $12 \%$ a los dos años y asciende hasta el $50 \%$ en el largo plazo. Como lo predice la teoría y lo muestran las estimaciones, la inflación aumenta y la política monetaria reacciona de manera acorde, es decir, aumenta la tasa de interés de política.

Ahora, ¿qué ocurre ante una perturbación de la política monetaria? El grado de transmisión es similar, en trayectoria y nivel, al que resulta de una perturbación exógena de la tasa de cambio; pero en este caso el peso se aprecia y el nivel de precios y el PIB disminuyen. Es decir, los impactos macroeconómicos de la política monetaria se retroalimentan y reducen el IPC.

¿Y ante las perturbaciones externas? En todos los casos la transmisión es positiva, entre $1 \%$ y $25 \%$, y estadísticamente significativa en el corto plazo, por lo menos para un intervalo de confiabilidad del $68 \%$. En el mediano y largo plazos es negativa en niveles entre $5 \%$ y $25 \%$, pero no es estadísticamente significativa después del cuarto año, en particular ante la perturbación del precio del petróleo.

Dada la importancia económica del comportamiento de los precios del petróleo para Colombia, vale la pena analizar en detalle el impacto macroeconómico de su perturbación. Como se observa, dicha perturbación aprecia de manera persistente la tasa de cambio, por lo menos hasta los dos primeros años, y aumenta la inflación, aunque en menor grado y a pesar del ajuste de 
Figura 2. Grado de transmisión de la tasa de cambio sobre el IPC

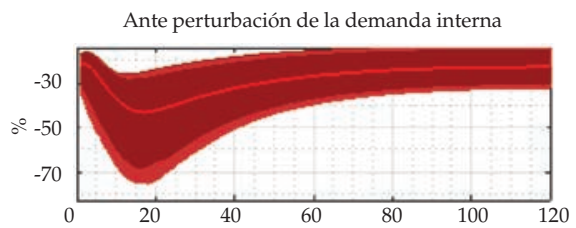

Ante perturbación de la oferta interna
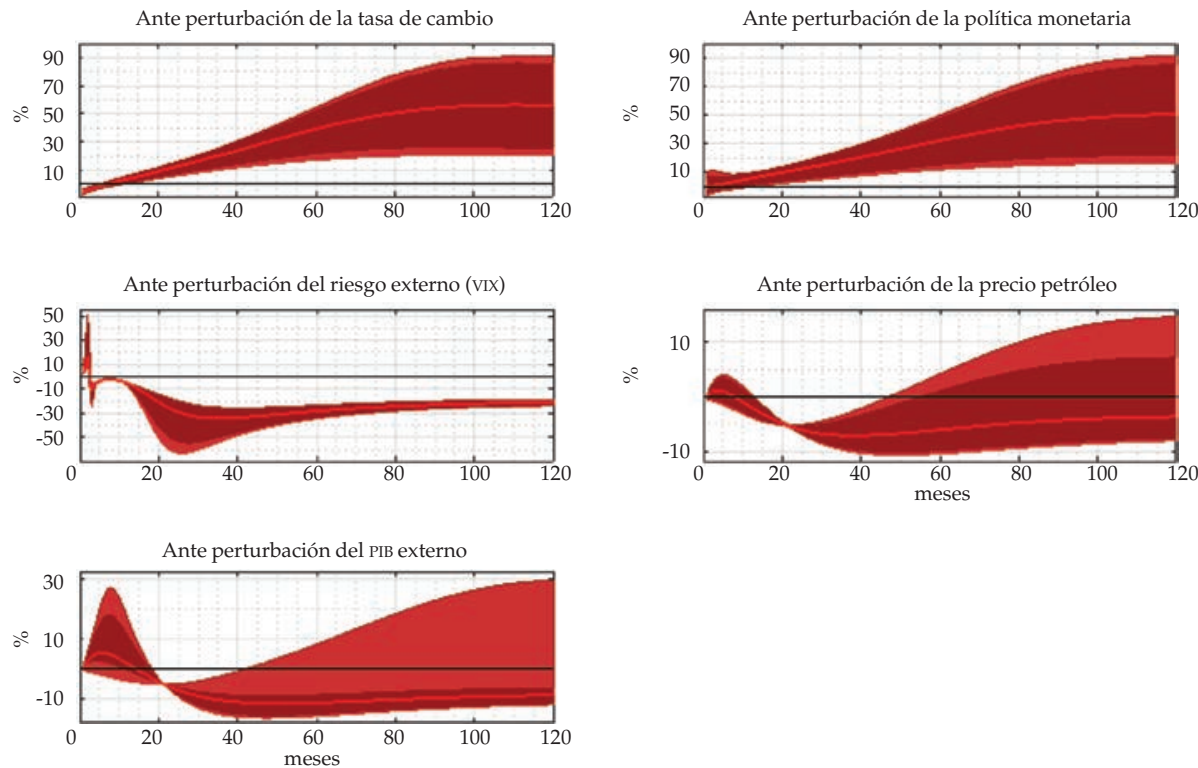

Fuente: cálculos de los autores.

la política monetaria. ¿Qué explica este resultado? El comportamiento positivo de la demanda agregada, que más que compensa el efecto negativo de la apreciación sobre el IPC, como se discutió en la sección anterior.

En conclusión, la transmisión de los movimientos de la tasa de cambio sobre los precios es perturbación-dependiente. En otras palabras, los movimientos de la tasa de cambio impactan los precios en una magnitud que depende del tipo de perturbación que cause dichos movimientos. Este resultado corrobora lo encontrado por Shambaugh (2008), para una muestra de países desarrollados y emergentes, entre ellos Colombia, y por Forbes et al. (2015), para el caso del Reino Unido. En segundo lugar, en todos los casos la transmisión acumulada es altamente persistente, incluso va más allá de los cinco años. Tercero, la mayor transmisión durante el horizonte de política monetaria - entre el primer mes y los siguientes dos años desde el momento de la perturbación - se observa ante una perturbación de la demanda interna. 
Esta más que compensa el impacto negativo sobre el IPC de la apreciación y de la reacción de la política monetaria que, al final, aumenta.

La implicación de política más importante de la anterior conclusión es inmediata: el grado de transmisión de movimientos de la tasa de cambio sobre el IPC depende del tipo de perturbación que los cause. El corolario es que no debería existir una regla específica sobre el grado de transmisión cambiaria sobre la inflación para la toma de decisiones de política monetaria.

\section{Descomposición histórica de la variación de tasa de cambio y la inflación}

Esta sección presenta y analiza la descomposición histórica (HD) de las perturbaciones para la variación de la tasa de cambio y la inflación del IPC. El objetivo es identificar las perturbaciones que originaron dichas variaciones a lo largo del tiempo y cuantificar su grado de participación. Este ejercicio permite ilustrar, de otra manera, la endogeneidad de los movimientos de la tasa de cambio y su impacto sobre el grado de su transmisión sobre los precios.

Recuerde que la HD es una aproximación a la magnitud de la contribución de cada perturbación al valor no predicho de cada una de las variables endógenas en el sistema SVAR, para cada momento a lo largo de la muestra. Es decir, la HD permite diferenciar qué perturbaciones fueron las principales determinantes del comportamiento de las variables endógenas del sistema ${ }^{12}$. La interpretación de la HD es la siguiente: un valor positivo de una perturbación cualquiera del sistema en el momento $t$ significa que dicha perturbación influyó positivamente sobre la variable endógena en dicho momento del tiempo. Si el valor es negativo, la interpretación es la contraria. Por tanto, la HD es una herramienta rigurosa y valiosa que permite revelar y valorar las perturbaciones que generan los movimientos de las variables macroeconómicas de las variables de interés.

Las figuras 3 y 4 muestran las descomposiciones históricas de la variación anual de tasa de cambio y la inflación anual del IPC, respectivamente. El tamaño de las barras mide la participación de la perturbación i-ésima en la explicación del comportamiento de la variable endógena analizada. Las zonas sombreadas de rosado (oscuro) corresponden a los siguientes periodos de depreciaciones del peso: entre abril de 2002 y marzo de 2003: 18 \%; entre

12 ¿Cuáles son las diferencias más importantes entre la descomposición de varianza, un ejercicio estándar en modelos de series de tiempo tipo VAR, y la descomposición histórica? Rincón-Castro y Rodríguez-Niño (2016) discuten algunas. 
febrero de 2006 y junio de 2006: $13 \%$; entre junio de 2008 y febrero de 2009: $31 \%$ y entre julio de 2014 y febrero de 2016: 60\%. Las zonas sombreadas de gris (claro) corresponden a los siguientes instantes de apreciaciones del peso: entre marzo 2003 y febrero de 2006: -20\%; entre junio de 2006 y junio de 2008: -28\%; entre febrero de 2009 y junio de 2012: -30\% y entre febrero y junio de 2016: $-11 \%$.

La figura 3 muestra la HD de la variación anual de tasa de cambio del peso, representada por la línea sólida negra, luego de restarle la media a la serie observada. En primer lugar, las perturbaciones del precio del petróleo y exógenas de la tasa de cambio y el PIB externo explican en buena medida su comportamiento a lo largo de la muestra.

Como lo indica la figura, son claras las presiones a la baja desde el año 2003, año en que se inició el boom de los precios de los productos básicos, entre ellos del petróleo. Sin embargo, hacia mediados de la muestra, la perturbación de los precios del petróleo actuó sobre el peso, pero en sentido contrario, ya que sufrieron una fuerte caída ante la profundización de la crisis financiera internacional por el anuncio de la quiebra de Lehman-Brothers el 15 de septiembre de 2008. Nótese que la crisis se manifestó en una depreciación rápida y fuerte del peso, a posteriori, de naturaleza temporal ${ }^{13}$. Por otro lado, en este mismo periodo la tasa de interés de política y el PIB externo presionaron el peso en sentido contrario, es decir, hacia la apreciación. Inmediatamente después, estas últimas perturbaciones actuaron en sentido contrario.

En la última parte de la muestra, la caída del precio del petróleo y la perturbación autónoma de la tasa de cambio se reflejan en una depreciación histórica del peso, tanto por su magnitud como por su carácter persistente. Vale resaltar que la perturbación negativa de la oferta interna durante este lapso aparece también como un determinante central de la depreciación.

La figura 4 muestra la HD de la inflación, representada por la línea sólida negra, luego de restarle la media a la serie observada. A diferencia de la tasa de cambio, aquí no es posible extraer una conclusión general sobre cuáles perturbaciones fueron las más importantes en explicar su comportamiento a lo largo de la muestra, como fue el caso de la tasa de cambio.

Entre el comienzo de la muestra y mediados de 2006, las perturbaciones de la política monetaria, del precio del petróleo, del riesgo país y el pIB externo presionaron la inflación al alza. Por el contrario, el movimiento autónomo de la tasa de cambio, la demanda y, en menor grado, la oferta la presionaron a la baja.

13 Recuérdese que la "tasa de cambio" en este documento alude a la tasa de cambio nominal efectiva del peso. En este mismo periodo la tasa representativa del mercado se depreció $47 \%$. 


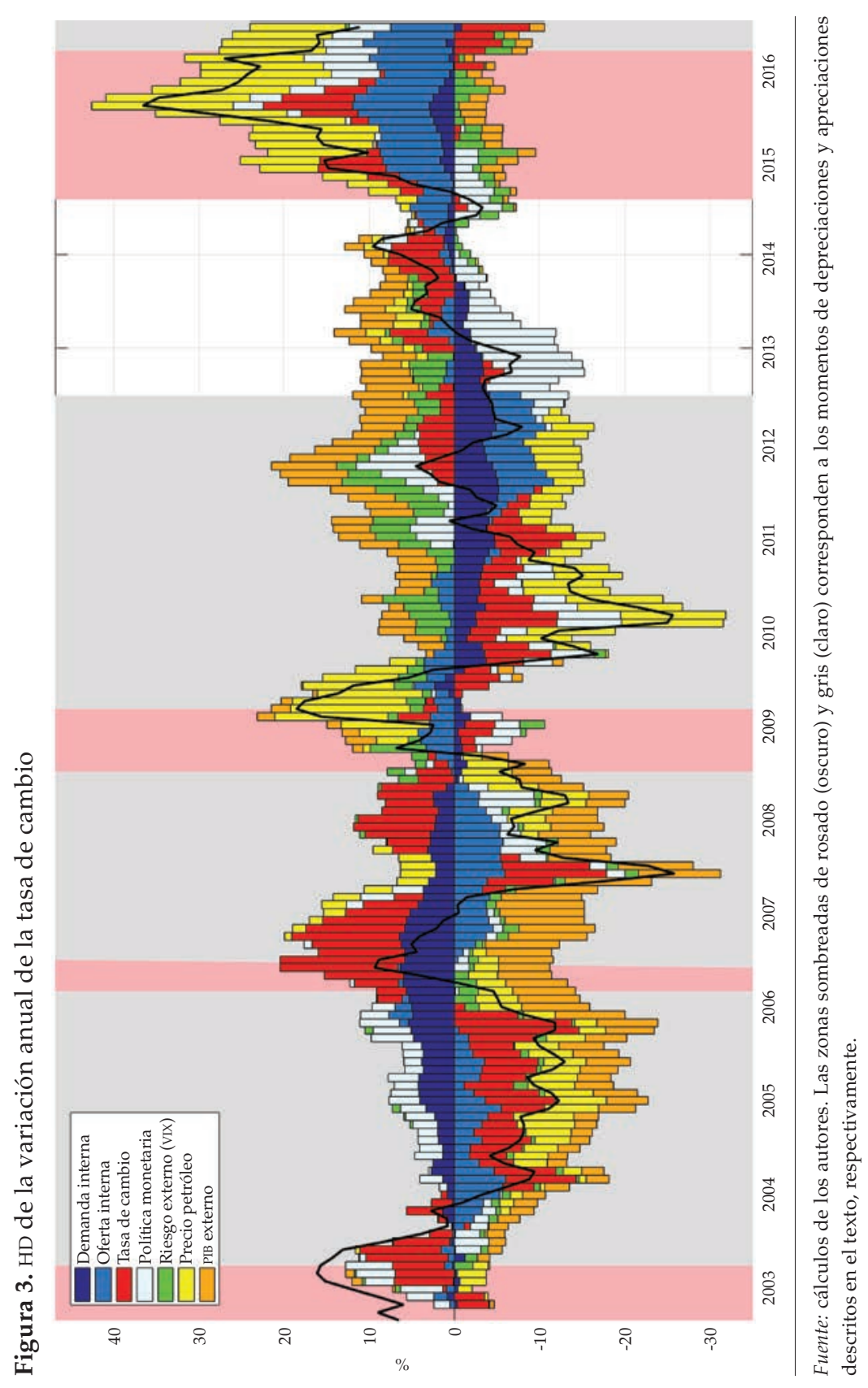




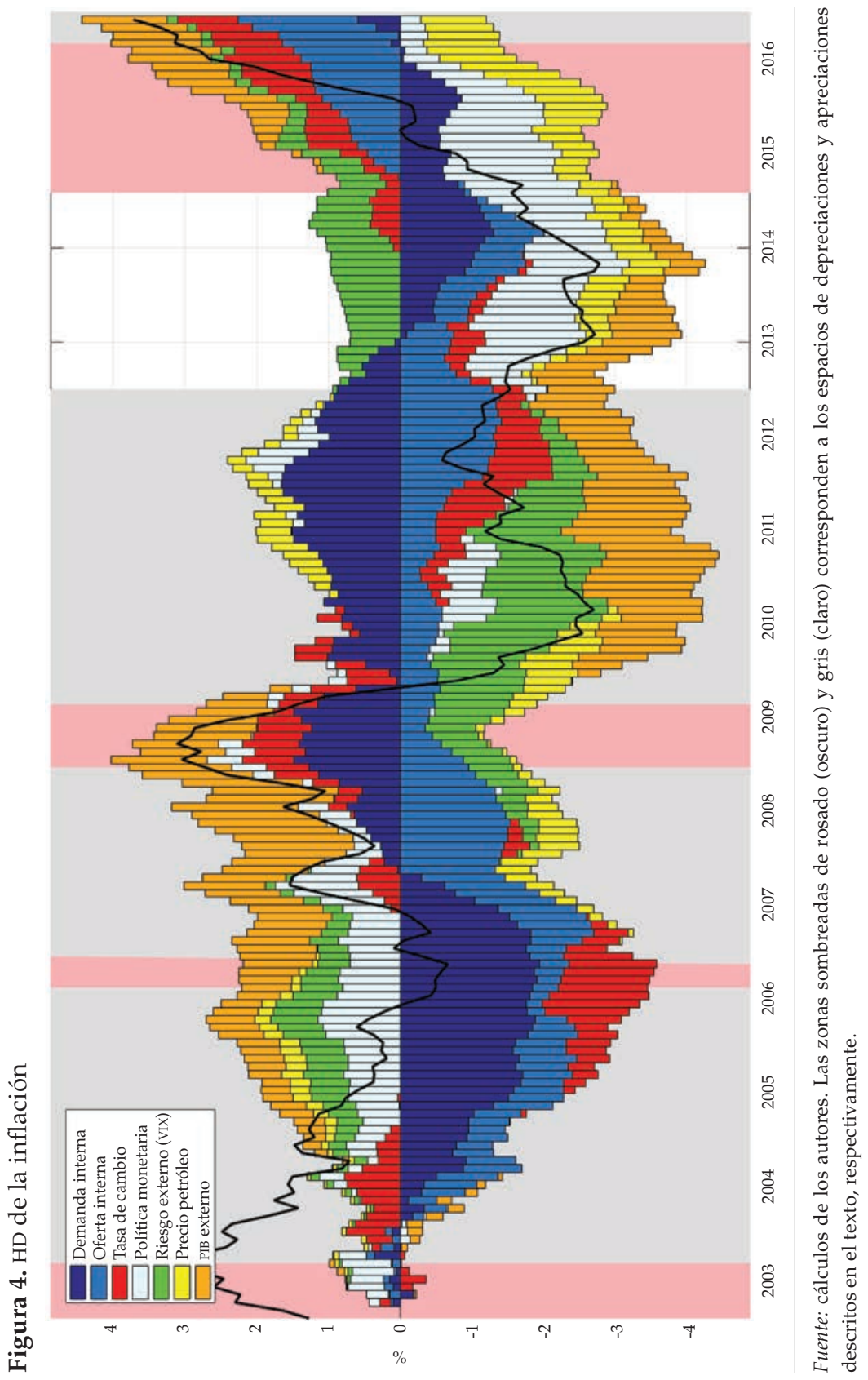


Como lo muestra la figura, estas últimas perturbaciones dominaron, ya que la inflación cayó a lo largo del periodo. Luego la inflación experimentó una fase de aumentos y disminuciones, pero con tendencia positiva, que culminó con una desviación positiva del 7,6\% en octubre de 2008, la tasa de inflación más alta desde septiembre de 2001. Las perturbaciones que presionaron la inflación al alza, y de manera significativa durante el momento, fueron la tasa de cambio, la demanda local y el PIB externo. Las perturbaciones de política monetaria también aparecen como impulsores de su incremento durante este periodo. Las perturbaciones que presionaron la inflación a la baja fueron la oferta interna, el riesgo internacional y, en menor media, el precio del petróleo.

Posterior a octubre de 2008, la inflación experimentó un lapso de continuo descenso, que la ubicó, inclusive, por debajo del rango meta ( $2 \%-4 \%)$. Excepto por el impacto positivo de la demanda hasta el año 2013, y del riesgo internacional entre 2013 y 2015, las demás perturbaciones mantuvieron bala la inflación durante este periodo.

A partir de febrero de 2015, la historia cambia de manera drástica, porque la inflación emprende una senda creciente y acelerada. Tres perturbaciones sobresalen en la explicación de esta tendencia ascendente, la oferta, la tasa de cambio y el PIB externo. En menor grado aparecen las presiones provenientes del riesgo externo. Por su parte, la demanda, la política monetaria y el precio del petróleo presionan la inflación a la baja ${ }^{14}$.

En resumen, las perturbaciones del precio del petróleo y las autónomas de la tasa de cambio y el PIB externo explican, en gran medida, el comportamiento de la tasa de cambio del peso a lo largo de la muestra. En cuanto a la inflación, es difícil establecer una conclusión general. Lo que sí se puede afirmar es que, de acuerdo con los resultados, la senda creciente y acelerada de la inflación del último momento de la muestra se explica, principalmente, por las perturbaciones de la oferta, de la tasa de cambio y del PIB externo.

\section{Conclusiones}

La literatura local e internacional que ha estudiado la transmisión de los movimientos de la tasa de cambio sobre los precios asume que aquellos son

14 Como complemento de los resultados de las figuras 3 y 4, el apéndice A.3 muestra la descomposición de varianza de la variación anual de la tasa de cambio y de la inflación. En adición, los apéndices A.4 y A.5 reportan resultados de las HDs de estas mismas dos variables para movimientos "grandes" y "pequeños" de la tasa de cambio y para las depreciaciones vis a vis apreciaciones. 
exógenos a las perturbaciones que impactan la economía o a ella misma. Sin embargo, este supuesto de exogeneidad ha sido puesto en duda recientemente por la literatura teórica y empírica. Dada la importancia de esta predicción para el análisis y la toma de decisiones de política monetaria, este documento estudia empíricamente dicha conjetura. Para cumplir su objetivo, el documento utiliza datos mensuales de Colombia para el periodo 2001-2016 y un modelo VAR estructural lineal. Los resultados muestran que el grado de transmisión depende de la perturbación que origine el movimiento de la tasa de cambio, es decir, que es perturbación-dependiente.

La implicación de política más importante del resultado es inmediata: el grado de transmisión de movimientos de la tasa de cambio sobre el IPC depende del tipo de perturbación que los cause. El corolario es que no debería existir una regla específica sobre el grado de transmisión para la toma de decisiones de política monetaria.

Por su lado, las HDs indican que las perturbaciones del precio del petróleo y las autónomas de la tasa de cambio y el PIB externo explican, en gran medida, el comportamiento de la tasa de cambio del peso a lo largo de la muestra. En cuanto a la inflación, es difícil establecer una conclusión general. Lo que sí se puede afirmar es que, de acuerdo con los resultados, la senda de la inflación del último año y medio se explican por perturbaciones de la oferta interna, de la tasa de cambio y del PIB externo.

Una posible extensión del presente documento es ampliar la muestra a un grupo de países avanzados y emergentes. Otra es estimar el modelo mediante técnicas de coeficientes cambiantes en el tiempo, en vez de coeficientes fijos, como el presentado y estimado aquí. Esa metodología permitiría capturar los cambios en la relación entre las variables endógenas y las perturbaciones; por tanto, en el grado de transmisión en el tiempo.

\section{Referencias}

Binning, A. (2013). Underidentified SVAR models: A framework for combining short and long-run restrictions with sign-restrictions. Working Paper, 14, Norges Bank.

Blanchard, O., \& Quah, D. (1989). The dynamic effects of aggregate demand and supply disturbances. American Economic Review, 79(4), 655-673.

Blanchard, O., \& Gali, J. (2007). The Macroeconomic Effects of Oil Shocks: Why are the 2000s so different from the 1970s? NBER Working Paper Series, No. 13368. 
Bonaldi, P., González, A., \& Rodríguez, D. (2010). Importancia de las Rigideces Nominales y Reales en Colombia: un enfoque de equilibrio general dinámico y estocástico. Borradores de Economía, 591, Banco de la República.

Canova, F., \& De Nicolo, Y. (2002). Monetary disturbances matter for business fluctuations in G-7. Journal of Monetary Economics, 49, 1131-1159.

Faust, J., \& Rogers, J. (2003). Monetary policy's role in exchange rate behavior. Journal of Monetary Economics, 50(7), 1403-1424.

Forbes, K., Hjortsoe, I., \& Nenova, T. (2015). The Shocks Matter: New Evidence on Exchange Rate Pass-Through. Discussion Paper 43, Bank of England.

Fry, R., \& Pagan, A. (2011). Sign Restrictions in Structural Vector Autoregressions: A Critical Review. Journal of Economic Literature, 49(4), 938-960.

Gali, J. (1999). Technology, Employment, and the Business Cycle: Do Technology Shocks Explain Aggregate Fluctuations? American Economic Review, 88(1), 249-271.

Kilian. L. (2009). Not All Oil Price Shocks Are Alike: Disentangling Demand and Supply Shocks in the Crude Oil Market. American Economic Review, 99(3), 1053-1069.

López, E., Montes, E., Garavito, A., \& Collazos, M. (2013). La economía petrolera en Colombia. En H. Rincón \& A. Velasco (eds.), Flujos de capitales, choques externos y respuestas de politica en países emergentes (pp. 337-407). Bogotá: Banco de la República.

Ouliaris, S., Pagan, A., \& Restrepo, J. (2016). Quantitative macroeconimic modeling with structural vector autoregressions- an e-Views implementation. Washington D.C.: IMF Institute for Capacity Development.

Rincón-Castro, H., \& Rodríguez-Niño, N. (2016). Pass-Through of Exchange Rate Shocks on Inflation: A Bayesian Smooth Transition VAR Approach. Borradores de Economía, 930, Banco de la República.

Rubio-Ramírez, J., Waggoner, D., \& Zha, T. (2010). Structural Vector Autoregressions: Theory of Identification and Algorithms for Inference. Review of Economic Studies, 77(2), 665-696.

Shambaugh, J. (2008). A new look at pass-through. Journal of International Money and Finance, 27(4), 560-591. 


\section{Apéndice A.1. Datos, fuentes y notas metodológicas}

Muestra: enero de 2001 - junio de 2016

Frecuencia: Mensual

- IPC: Índice de precios al consumidor. Fuente: Banco de la República.

- E: Índice de tasa de cambio nominal efectiva (pesos/USD). Se pondera por comercio total utilizando las participaciones de los principales socios comerciales de Colombia: Estados Unidos de América, Canadá, Japón, Reino Unido, Alemania, Holanda, España, Francia, Italia, Bélgica, Suiza, Suecia, México, Panamá, Venezuela, Ecuador, Perú, Brasil, Chile, Argentina. Fuente: Banco de la República (estadísticas no publicadas).

- PIB: Producto Interno Bruto real trimestral de Colombia. Para obtener la serie mensual se interpola la serie trimestral mediante la serie mensual del Indicador de Seguimiento a la Economía (ISE) del Dane y el procedimiento "DISAGGREGATE.SRC" del programa econométrico RATS. Fuente: Banco de la República (estadísticas no publicadas) y cálculos propios.

- тів: Tasa de interés interbancaria. Fuente: Banco de la República.

- vIX: Índice de volatilidad del mercado de opciones de Chicago. Los datos mensuales son el promedio de los datos diarios. Fuente: Datastream.

- WTI: West Texas Intermediate, precio de cotización del petróleo WTI. Datos mensuales calculados como el promedio de los datos diarios. La serie se deflacta mediante el Índice de Precios al Productor de los Estados Unidos. Fuente: Datastream y cálculos propios.

- PIB externo: Producto Interno Bruto real mensual de los Estados Unidos de América. Se utiliza como proxy la serie "Chicago FED National Activity Index, CFNAI-MA3". Fuente: Federal Reserve Bank of Chicago. 
Apéndice A.2. Funciones de impulso respuesta de las variables endógenas ante su misma perturbación
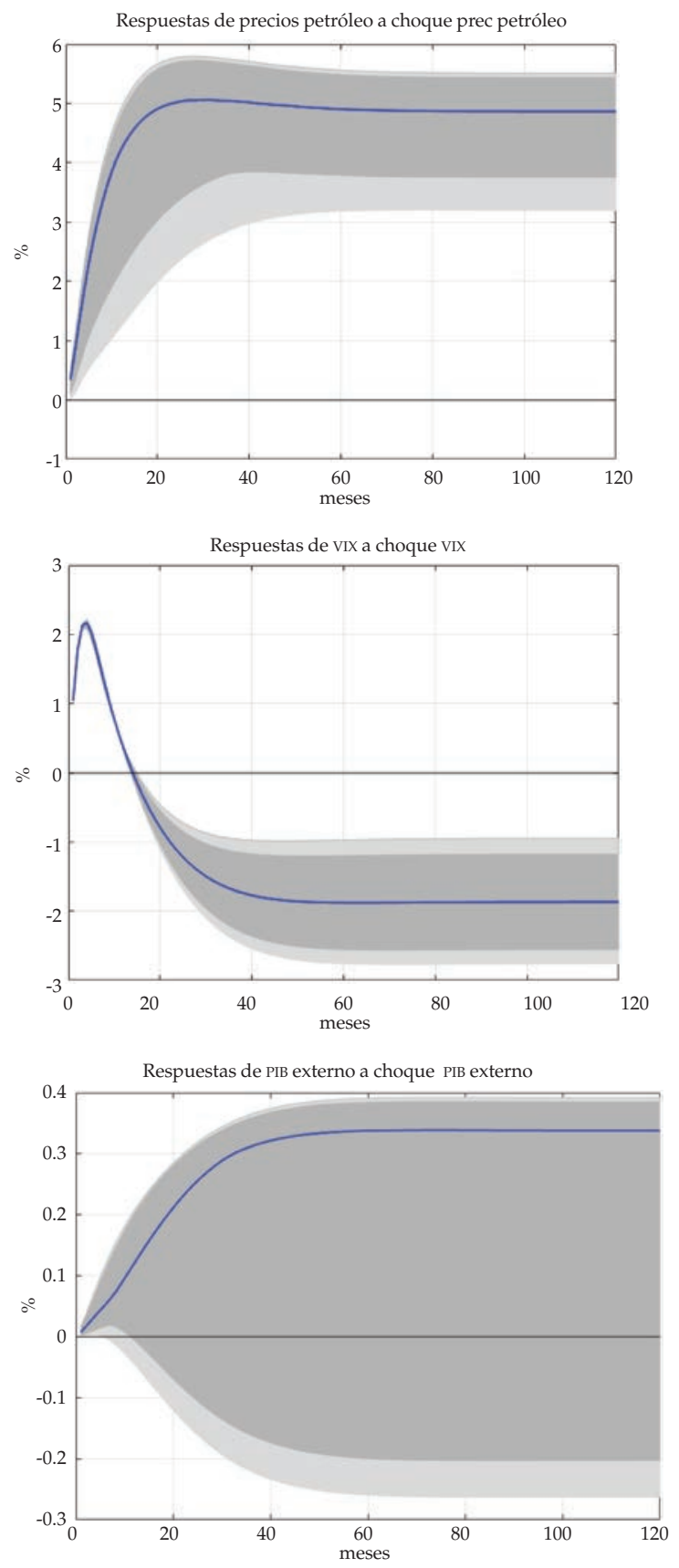


\section{Apéndice A.3. Descomposición de varianza}

\begin{tabular}{lccccccccc|}
\hline \multicolumn{7}{c}{ Proporción de la varianza explicada por perturbación a: } \\
\hline Variable & $\begin{array}{c}\text { Horizonte } \\
\text { (meses) }\end{array}$ & Demanda & Oferta & $\begin{array}{c}\text { Tasa de } \\
\text { cambio }\end{array}$ & $\begin{array}{c}\text { Política } \\
\text { monetaria }\end{array}$ & VIX & $\begin{array}{c}\text { Precio del } \\
\text { petróleo }\end{array}$ & $\begin{array}{c}\text { PIB ex- } \\
\text { terno }\end{array}$ \\
\hline $\begin{array}{l}\text { Variación anual } \\
\text { del IPC }\end{array}$ & 1 & 25 & 1 & 32 & 0 & 28 & 8 & 6 \\
\hline $\begin{array}{l}\text { Variación anual } \\
\text { del IPC }\end{array}$ & 12 & 1 & 7 & 36 & 19 & 1 & 22 & 15 \\
\hline $\begin{array}{l}\text { Variación anual } \\
\text { del IPC }\end{array}$ & 24 & 2 & 8 & 39 & 20 & 3 & 21 & 8 \\
\hline $\begin{array}{l}\text { Variación anual de } \\
\text { la tasa de cambio }\end{array}$ & 1 & 2 & 4 & 25 & 12 & 23 & 31 & 4 \\
\hline $\begin{array}{l}\text { Variación anual de } \\
\text { la tasa de cambio }\end{array}$ & 12 & 1 & 2 & 12 & 6 & 19 & 60 & 1 \\
\hline $\begin{array}{l}\text { Variación anual de } \\
\text { la tasa de cambio }\end{array}$ & 24 & 1 & 2 & 11 & 5 & 21 & 60 & 1 \\
\hline
\end{tabular}

Fuente: cálculos de los autores.

\section{Apéndice A.4. Participación porcentual de las perturbaciones macroeconómicas en el comportamiento histórico de la tasa de cambio y la inflación, condicionada a los periodos de apreciaciones y depreciaciones del peso descritas en el texto}

\begin{tabular}{ccccccccc}
\multicolumn{7}{c}{ Variación anual de la tasa de cambio } \\
\hline \multicolumn{7}{c}{ Perturbaciones } \\
\hline $\begin{array}{c}\text { Fecha } \\
\text { inicial }\end{array}$ & $\begin{array}{c}\text { Fecha } \\
\text { final }\end{array}$ & Demanda & Oferta & $\begin{array}{c}\text { Tasa de } \\
\text { cambio }\end{array}$ & $\begin{array}{c}\text { Política } \\
\text { monetaria }\end{array}$ & VIX & $\begin{array}{c}\text { Precio del } \\
\text { petróleo }\end{array}$ & PIB Externo \\
\hline 2002:04 & $2003: 03$ & 4 & 7 & 36 & 33 & 4 & 13 & 5 \\
\hline 2003:04 & $2006: 02$ & 11 & 13 & 27 & 13 & 4 & 15 & 18 \\
\hline 2006:03 & $2006: 06$ & 22 & 1 & 32 & 3 & 6 & 12 & 25 \\
\hline 2006:07 & $2008: 06$ & 11 & 12 & 26 & 8 & 4 & 12 & 26 \\
\hline 2008:06 & $2009: 02$ & 5 & 13 & 13 & 9 & 12 & 32 & 16 \\
\hline 2009:02 & $2012: 06$ & 13 & 10 & 15 & 11 & 11 & 25 & 16 \\
\hline 2014:07 & $2016: 02$ & 4 & 23 & 12 & 11 & 6 & 38 & 6 \\
\hline 2016:02 & $2016: 06$ & 2 & 25 & 16 & 15 & 4 & 34 & 5 \\
\hline
\end{tabular}

Fuente: cálculos de los autores con base en los resultados de las HDs presentadas en el texto. Los valores tabulados corresponden al promedio de los valores absolutos de las participaciones de la respectiva perturbación en la HD de la variación anual de la tasa de cambio. Como dichos valores son participaciones, la suma horizontal es igual a 100. Las zonas sombreadas de rosado y gris corresponden a los periodos de depreciaciones y apreciaciones, respectivamente, tal y como se describen en el texto. 
Variación anual de la inflación

\begin{tabular}{ccccccccc}
\hline \multicolumn{10}{c}{ Perturbaciones } \\
\hline $\begin{array}{c}\text { Fecha } \\
\text { inicial }\end{array}$ & $\begin{array}{c}\text { Fecha } \\
\text { final }\end{array}$ & Demanda & Oferta & $\begin{array}{c}\text { Tasa de } \\
\text { cambio }\end{array}$ & $\begin{array}{c}\text { Política } \\
\text { monetaria }\end{array}$ & VIx & $\begin{array}{c}\text { Precio del } \\
\text { petróleo }\end{array}$ & PIB Externo \\
\hline 2002:04 & $2003: 03$ & 13 & 11 & 17 & 50 & 2 & 3 & 4 \\
\hline 2003:04 & $2006: 02$ & 26 & 17 & 18 & 15 & 8 & 5 & 11 \\
\hline 2006:03 & $2006: 06$ & 33 & 7 & 21 & 14 & 9 & 2 & 15 \\
\hline 2006:07 & $2008: 06$ & 18 & 24 & 7 & 11 & 6 & 7 & 28 \\
\hline 2008:06 & $2009: 02$ & 27 & 12 & 13 & 4 & 16 & 3 & 25 \\
\hline 2009:02 & $2012: 06$ & 20 & 15 & 10 & 6 & 20 & 7 & 22 \\
\hline 2014:07 & $2016: 02$ & 12 & 16 & 12 & 25 & 8 & 16 & 12 \\
\hline 2016:02 & $2016: 06$ & 5 & 30 & 16 & 6 & 3 & 18 & 22 \\
\hline
\end{tabular}

Fuente: cálculos de los autores con base en los resultados de las HDs presentadas en el texto. Los valores tabulados corresponden al promedio de los valores absolutos de las participaciones de la respectiva perturbación en la HD de la variación anual del IPC. Como dichos valores son participaciones, la suma horizontal es igual a 100. Las zonas sombreadas de rosado y gris corresponden a los periodos de depreciaciones y apreciaciones, respectivamente, tal y como se describen en el texto.

\section{Apéndice A.5. Participación porcentual de las perturbaciones macroeconómicas en el comportamiento histórico de la tasa de cambio y la inflación, condicionada a periodos de movimientos "grandes" y "pequeños" de la tasa de cambio y a las apreciaciones o depreciaciones del peso}

Variación anual de la tasa de cambio

\begin{tabular}{lccccccc}
\hline \multicolumn{7}{c}{ Perturbaciones } \\
\hline $\begin{array}{c}\text { Fluctuación } \\
\text { de la tasa de } \\
\text { cambio }\end{array}$ & Demanda & Oferta & $\begin{array}{c}\text { Tasa de } \\
\text { cambio }\end{array}$ & $\begin{array}{c}\text { Política } \\
\text { monetaria }\end{array}$ & VIX & $\begin{array}{c}\text { Precio del } \\
\text { petróleo }\end{array}$ & PIB Externo \\
\hline Grandes & 9 & 13 & 22 & 12 & 6 & 26 & 13 \\
Pequeñas & 11 & 13 & 19 & 15 & 9 & 12 & 21 \\
Apreciación & 12 & 12 & 19 & 13 & 7 & 17 & 19 \\
Depreciación & 7 & 14 & 22 & 15 & 7 & 22 & 13 \\
\hline
\end{tabular}

Fuente: cálculos de los autores con base en los resultados de las HDs presentadas en el texto. Los valores tabulados corresponden al promedio de los valores absolutos de las participaciones de la respectiva perturbación en la HD de la variación anual de la tasa de cambio. Como son participaciones, la suma horizontal es igual a 100. Las fluctuaciones cambiarias "Grandes" son aquellas que en valor absoluto son mayores al $7 \%$. Las "Pequeñas" como las menores a dicho umbral. Este corresponde a la mediana de la distribución de la variación de anual de la tasa de cambio. En este cuadro "Apreciación" y "Depreciación" indican que la variación de la tasa de cambio es negativa y positiva, respectivamente. 
Variación anual de la inflación

\begin{tabular}{lccccccc}
\hline \multicolumn{7}{c}{ Perturbaciones } \\
\hline $\begin{array}{c}\text { Fluctuación } \\
\text { de la tasa de } \\
\text { cambio }\end{array}$ & Demanda & Oferta & $\begin{array}{c}\text { Tasa de } \\
\text { cambio }\end{array}$ & $\begin{array}{c}\text { Política mo- } \\
\text { netaria }\end{array}$ & VIX & $\begin{array}{c}\text { Precio del } \\
\text { petróleo }\end{array}$ & PIB Externo \\
\hline Grandes & 19 & 17 & 11 & 16 & 12 & 9 & 17 \\
Pequeñas & 21 & 16 & 13 & 15 & 12 & 6 & 18 \\
Apreciación & 21 & 18 & 10 & 12 & 13 & 6 & 20 \\
Depreciación & 18 & 15 & 14 & 19 & 11 & 10 & 14 \\
\hline
\end{tabular}

Fuente: cálculos de los autores con base en los resultados de las HDs presentadas en el texto. Los valores tabulados corresponden al promedio de los valores absolutos de las participaciones de la respectiva perturbación en la HD de la variación anual del IPC. Como son participaciones, la suma horizontal es igual a 100. Las fluctuaciones cambiarias "Grandes" son aquellas que en valor absoluto son mayores al $7 \%$. Las "Pequeñas" como las menores a dicho umbral. Este corresponde a la mediana de la distribución de la variación de anual de la tasa de cambio. En este cuadro "Apreciación" y “Depreciación" indican que la variación de la tasa de cambio es negativa y positiva, respectivamente. 
